\title{
Recurrent Oxaliplatin-Induced Immune Hemolytic Anemia by Chemotherapy for Metastatic Colon Cancer: A Case Report ${ }^{*}$
}

\author{
Sandrine Oziel-Taieb, Renaud Sabatier, Marine Gilabert, Sylvain Garciaz, Pauline Ries, \\ Slimane Dermeche, Jean-Luc Raoul ${ }^{\#}$
}

Department of Medical Oncology, Paoli-Calmettes Institute, Marseille, France.

Email: " raoulj1@ipc.unicancer.fr

Received December $12^{\text {th }}, 2012$; revised January $14^{\text {th }}, 2013$; accepted January $22^{\text {nd }}, 2013$

\begin{abstract}
We report herein the case of a 55-year-old man who presented an acute hemolysis following a 6th cycle of FOLFOX regimen for a metastatic colorectal cancer; this patient had previously received 12 cycles in an adjuvant setting. He had presented attenuated signs during the previous cycle. This corresponded to an oxaliplatin-induced immune hemolytic anemia. This unfrequent side effect of oxaliplatin needs to be known in order to avoid severe complications.
\end{abstract}

Keywords: Oxaliplatine; Hemolysis; Auto-Immune; Colorectal Cancer

\section{Introduction}

Oxaliplatin is used in the treatment of colorectal cancer in adjuvant or metastatic setting. The side effects are generally moderate: hypersensitivity, digestive disorders, hematological toxicity, acroparesthesia exacerbated by the cold. More rarely, oxaliplatin can be responsible for immune-induced cytopenia that can sometimes threaten the vital prognosis. We report a rare case of a patient presenting with autoimmune hemolytic anemia induced by oxaliplatin after several months of treatment, with rapid and favorable resolution.

\section{Clinical Case}

A 55-year-old patient treated for metastatic colon cancer was admitted for the 5th session of FOLFOX treatment. His only medical history was allergic asthma treated by formoterol and beclometasone.

In December 2009, the patient was operated on for adenocarcinoma of the upper rectum classified as pT3N2M0, with a mutation on KRAS. He received adjuvant chemotherapy with FOLFOX (12 sessions) between January and June 2010 without toxicity other than resolving grade 1 neuropathy. In February 2011, hepatic and lymph nodes progression led to further chemotherapy with a FOLFIRI-bevacizumab regimen, followed by FOLFIRI alone from May to August 2011 for a venous

*None of the authors has a conflict of interest in relation with this topic.

\#Corresponding author. thrombotic incident. Partial remission was obtained and capecitabin was proposed as maintenance therapy from October 2011 to January 2012 until hepatic progression indicating re-introduction of the FOLFOX regimen.

On the last day of the 5th session, questioning the patient revealed that he had suffered from shivering and colored urine at home a few hours after oxaliplatin administration. The episode resolved rapidly without sequellae and there was no other clinical anomaly. Clinical examination of the patient before the 5th session was normal, with PS 0 and no blood test abnormality. Blood tests showed: hemoglobin $13.8 \mathrm{~g} / \mathrm{dL}$, platelets $330,000 /$ $\mathrm{mm}^{3}$ and creatininemia $97 \mu \mathrm{mol} / 1$.

The patient received the same chemotherapy doses as during previous cycles associated with hydration and premedication by $60 \mathrm{mg}$ of prednisolone. At the end of oxaliplatin infusion the patient suffered from lower back pain with hematuria and fever $\left(38.6^{\circ} \mathrm{C}\right)$. Infusion was stopped immediately and the patient was again given intravenous prednisolone $(120 \mathrm{mg})$. Blood tests performed during these symptoms showed: leukocytes $=$ $7100 / \mathrm{mm}^{3} ; \mathrm{Hb}=9.1 \mathrm{~g} / \mathrm{dL} ;$ platelets $=218,000 / \mathrm{mm}^{3}$; Bilirubin $=37.5 \mu \mathrm{mol} / \mathrm{L}$ (unconjugated $=19.5 \mu \mathrm{mol} / \mathrm{L}$ ); $\mathrm{LDH}=903 \mathrm{IU} / \mathrm{L}(\mathrm{N}<248 \mathrm{IU} / \mathrm{L}) ;$ Haptoglobin $=0.15$ $\mathrm{g} / \mathrm{L}(\mathrm{N}=0.36$ to $1.95 \mathrm{~g} / \mathrm{L})$; creatininemia $=95 \mu \mathrm{mol} / \mathrm{L}$. The Coombs erythrocyte test was positive (positive direct test with Anti-IgG antiglobulin; negative direct test with Anti-C3d antiglobulin).

The patient was hospitalized for monitoring, hydration 
and IV corticosteroid treatment (prednisolone $80 \mathrm{mg} \times$ 2/d). All clinical symptoms disappeared within $24 \mathrm{~h}$. Haptoglobin remained low for three days while anemia rapidly corrected itself without transfusion.

Immunoallergic hemolysis of oxaliplatin was strongly suspected and oxaliplatin was definitively contraindicated for this patient.

\section{Discussion}

In our case report, the sudden onset after oxaliplatin infusion of lower back pain associated with brown urine and a positive Coombs erythrocyte test, as well as increased LDH and reduced haptoglobin levels, suggested oxaliplatin induced intravascular hemolysis. The increasing severity and gravity of hemolysis between the last two chemotherapy sessions, the faster time to onset and worsening of symptoms led to concern that a major reaction might occur in case of re-administration of oxaliplatin. The rapid resolution of symptoms and correction of biological parameters was probably assisted by early administration of high doses of corticosteroids and hyperhydration.

Fifteen cases have been reported in the literature of which only the first was fatal [1]. The majority of cases concerned patients treated for metastatic colorectal cancer. One case occurred in the course of adjuvant chemotherapy [2]. This event has never been described in the course of a first administration of oxaliplatin. The earliest case occurred at the 4th session [3] but the patient had been receiving globular transfusions since the 2 nd session. Hemolysis always resolved after stopping administration of the molecule. In all cases, this concerned acute intravascular-type hemolysis with the following clinical diagnostic criteria: sudden onset a few minutes or hours after administration of oxaliplatin, lower back pain, fever, shivering and brown urine.

Association with more or less severe thrombopenia is not rare (8/15) and may require platelet transfusions. In around $50 \%$ of cases, there is also kidney failure with hemolytic and uremic syndrome of varying degrees of severity which could require hemodialysis $[4,5]$.

Biological features associated with this adverse event are a sudden drop in hemoglobin, drop in haptoglobin, increase in LDH and unconjugated bilirubin. The Coombs erythrocyte test continued to be positive for anti-IgG $+/-$ anti-Complement (contrary to hemolysis caused by the hemolytic and uremic syndrome). The mechanisms leading to the production of antibodies against red cells remain poorly understood. The most likely mechanism is the initiation of an immune response through interaction of the drug or one of its metabolites with the cell membrane of red blood cells, giving rise to a neoantigen and thus the production of drug-dependent antibodies and/or auto-antibodies [6]. More recently, another mechanism has been suspected with regard to platin derivative, including oxaliplatin, with cell membrane changes leading to non-immunological adsorption of proteins on the globular cell membrane and hemolysis triggered by the macrophage system. This mechanism is thus not thought to induce antibodies directed against the drug [7]. It is possible that these two mechanisms coexist in the case of oxaliplatin-induced hemolytic anemia.

In conclusion, it would appear to be necessary to recognize the existence of exceptional cases of intravascular hemolytic anemia linked to the use of oxaliplatin, a major active ingredient. The onset of a severe hemolytic episode does not appear to be predictable, and sometimes questioning the patient reveals self-limiting and spontaneously resolving symptoms during previous chemotherapy sessions (fever, lower back pain, brown urine).

The rapid administration of IV corticosteroids along with hyperhydration and medical monitoring can be essential factors in its management. The prognosis appears to be favorable, although in some cases considerable transfusion support as well as life-threatening acute kidney failure may require the use of resuscitation methods.

\section{REFERENCES}

[1] J. Desrame, H. Broustet, P. Darodes de Tailly, D. Girard and J. M. Saissy, "Oxaliplatin-Induced Haemolytic Anaemia,” Lancet, Vol. 354, No. 9185, 1999, pp. 1179-1180. doi:10.1016/S0140-6736(99)03827-1

[2] S. Buti, M. Riccò, M. D. Chiesa, B. Copercini, G. Tomasello, M. Brighenti and R. Passalacqua, "OxaliplatinInduced Hemolytic Anemia during Adjuvant Treatment of a Patient with Colon Cancer: A Case Report," Anticancer Drugs, Vol. 18, No. 3, 2007, pp. 297-300. doi:10.1097/CAD.0b013e3280102f4b

[3] J. I. Lai, W. S. Wang "Acute Hemolysis after Receiving Oxaliplatin Treatment: A Case Report and Literature Review," Pharmacy World \& Science, Vol. 31, No. 5, 2009, pp. 538-541. doi:10.1007/s11096-009-9313-0

[4] I. Ito, Y. Ito, M. Mizuno, Y. Suzuki, K. Yasuda, T. Ozaki, et al., "A Rare Case of Acute Kidney Injury Associated with Autoimmune Hemolytic Anemia and Thrombocytopenia after Long-Term Usage of Oxaliplatin," Clinical and Experimental Nephrology, Vol. 16, No. 3, 2012, pp. 490-494. doi:10.1007/s10157-012-0620-8

[5] A. Ulusakarya, S. Misra, M. Haydar, H. Habert, V. Castagne, Y. Gumus, et al., "Acute Renal Failure Related to Oxaliplatin-Induced Intravascular Hemolysis," Medical Oncology, Vol. 27, No. 4, 2010, pp. 1425-1426. doi:10.1007/s12032-009-9263-3

[6] A. Salama, "Drug-Induced Immune Hemolytic Anemia," Expert Opinion on Drug Safety, Vol. 8, No. 1, 2009, pp. 73-79. doi: $10.1517 / 14740330802577351$

[7] G. Garratty, "Drug-Induced Immune Hemolytic Anemia," Hematology ASH Education Program, Vol. 2009, No. 1, 2009, pp. 73-79. 